\title{
optica
}

\section{Correcting field-dependent aberrations with nanoscale accuracy in three-dimensional single-molecule localization microscopy: supplementary material}

\author{
Lexy von Diezmann, ${ }^{1}$ Maurice Y. Lee, ${ }^{1,2}$ Matthew D. LeW, ${ }^{1,3,4}$ And \\ W. E. MOERner ${ }^{1 *}$ \\ ${ }^{1}$ Department of Chemistry, Stanford University, Stanford, CA 94305 \\ ${ }^{2}$ Biophysics Program, Stanford University, Stanford, CA 94305 \\ ${ }^{3}$ Department of Electrical Engineering, Stanford University, Stanford, CA 94305 \\ ${ }^{4}$ Current address: Department of Electrical and Systems Engineering, Washington University in St. Louis, MO 63130 \\ *Corresponding author: wmoerner@stanford.edu \\ Published 19 November 2015
}

This document provides supplementary information to "Correcting field-dependent aberrations with nanoscale accuracy in three-dimensional single-molecule localization microscopy," http://dx.doi.org/10.1364/ optica.2.000985. This supplement consists of additional data in the form of supplementary figures. (C) 2015 Optical Society of America 
(a)

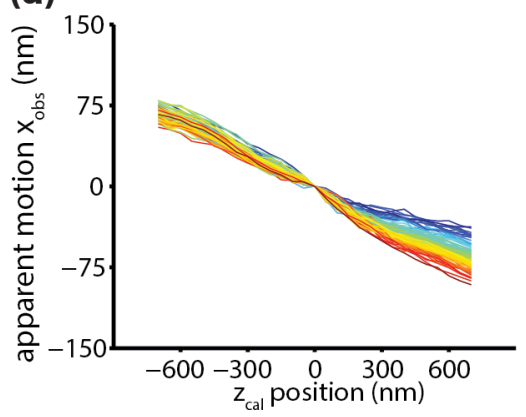

(d)

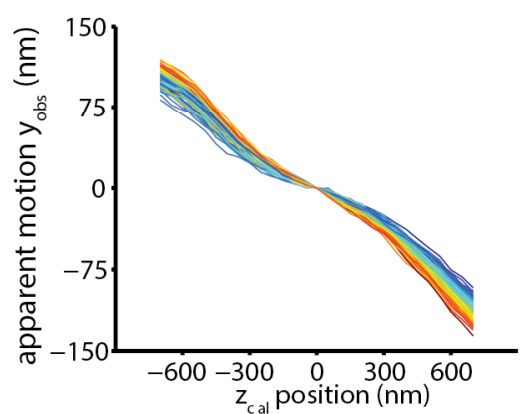

(b)

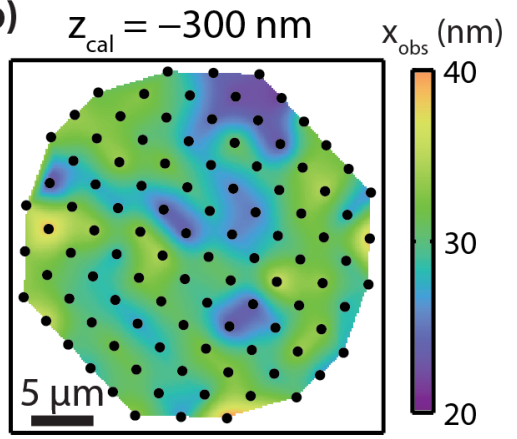

(e)

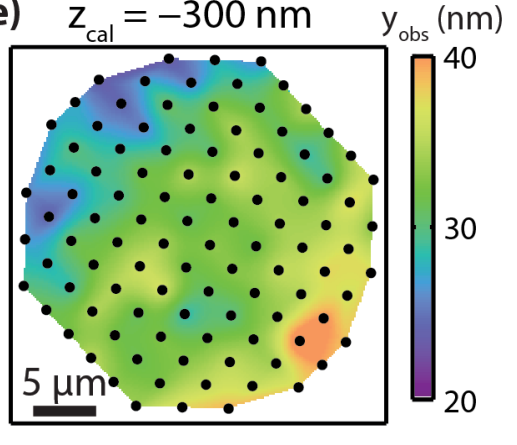

(c)

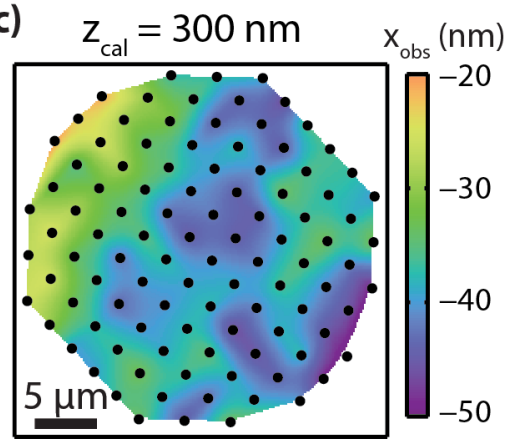

(f)

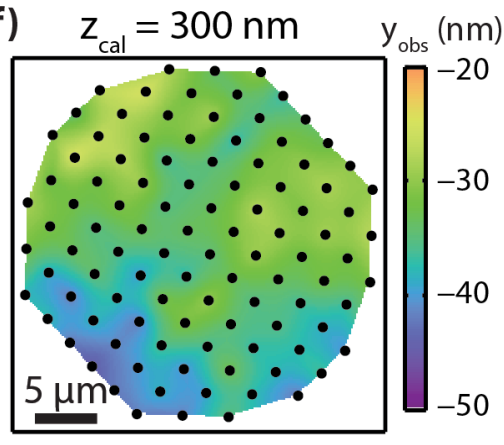

Figure S1. Field-dependent variation in the apparent $x y$ motion of the DH-PSF measured with the nanohole array (NHA). The apparent $x y$ position of the DH-PSF, defined as the midpoint between the two lobes as determined by a double-Gaussian fit, changes slightly as a function of $z$. We account for this during $z$ calibration by generating correction curves $x_{o b s}\left(z_{c a l}\right)$ and $y_{o b s}\left(z_{c a l}\right)$ that are subtracted from single-molecule localizations. (a), The calibration curves $x_{o b s}\left(z_{c a l}\right)$ collected from the NHA show a variety of behaviors, colored according to each curve's value of $x_{o b s}\left(z_{c a l}=700 \mathrm{~nm}\right)$. The field-dependence of the variation in apparent $x$ motion, $x_{o b s}$, is shown at $z_{c a l}=-300 \mathrm{~nm}$ (b) and $+300 \mathrm{~nm}(\mathrm{c})$. (d-f), the field-dependence for apparent $y$ motion $y_{o b s,}$ plotted as for $x_{o b s .}$ In actual experiments using a NHA, a local $x y$ correction derived from these data should be applied to the acquired data in a fashion similar to that for the z-correction described in the text. Note that the total magnitude of variation $(\sim 20 \mathrm{~nm})$ is substantially greater than the effective $x y$ localization precision of the holes at each $z_{c a l} \operatorname{step}(\sim 1 \mathrm{~nm})$. 
Total constant $z$ error

Initial calibration

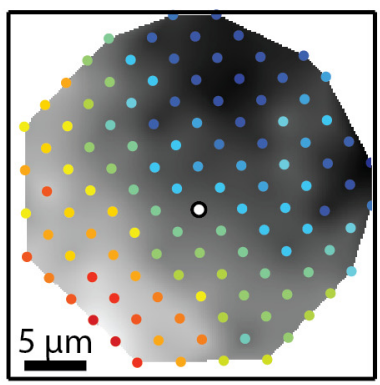

Rotate sample $180^{\circ}$

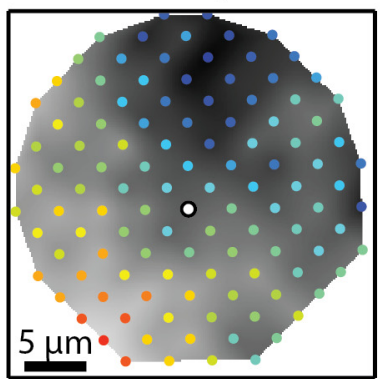

Tilt-corrected error

Initial calibration

70

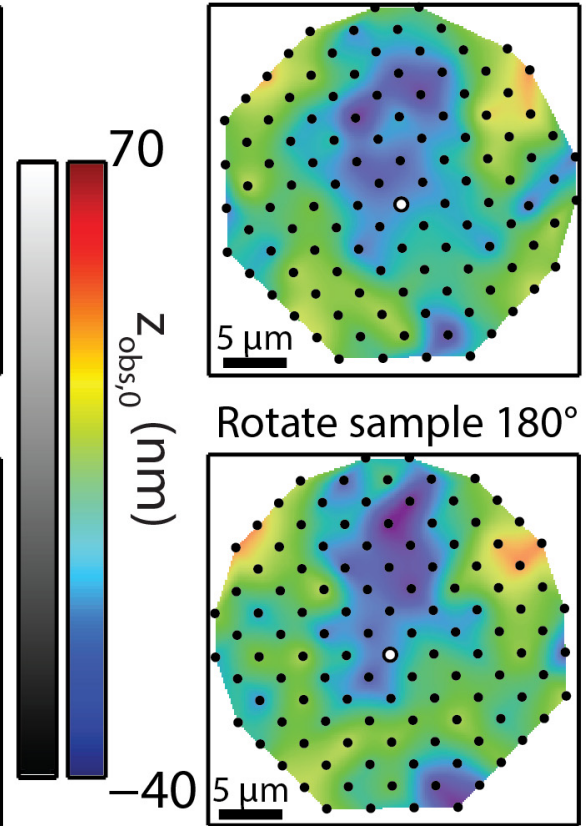

Response error, $\mathrm{z}_{\mathrm{cal}}=300 \mathrm{~nm}$ Initial calibration

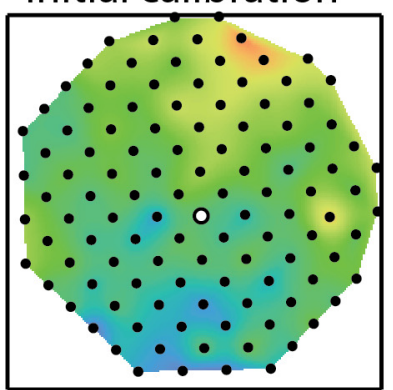

Rotate sample $180^{\circ}$
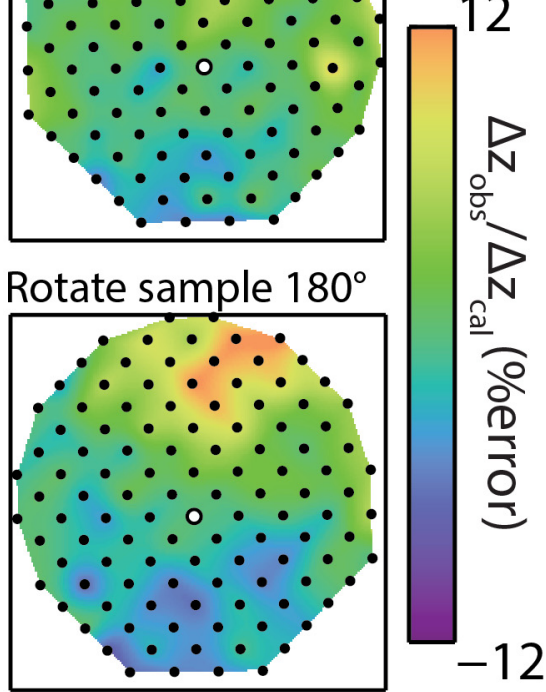

Figure S2. The results from NHA calibrations are not sensitive to the placement of nanoholes. Left column: the z offsets observed

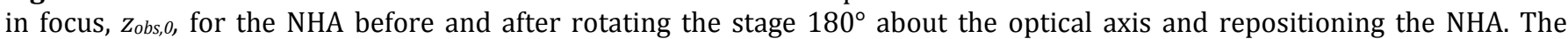
calibration nanohole is marked in white. Middle column: the residual errors after removing planar sample tilt, $z_{\text {res, }, 0}$, before and after rotation. Right column: the response errors $\left(\Delta z_{o b s}-\Delta z_{c a l}\right) / \Delta z_{c a l}$ before and after rotation. These data were acquired two months after the data in Figure 3, which accounts for a slight difference from those measurements. 

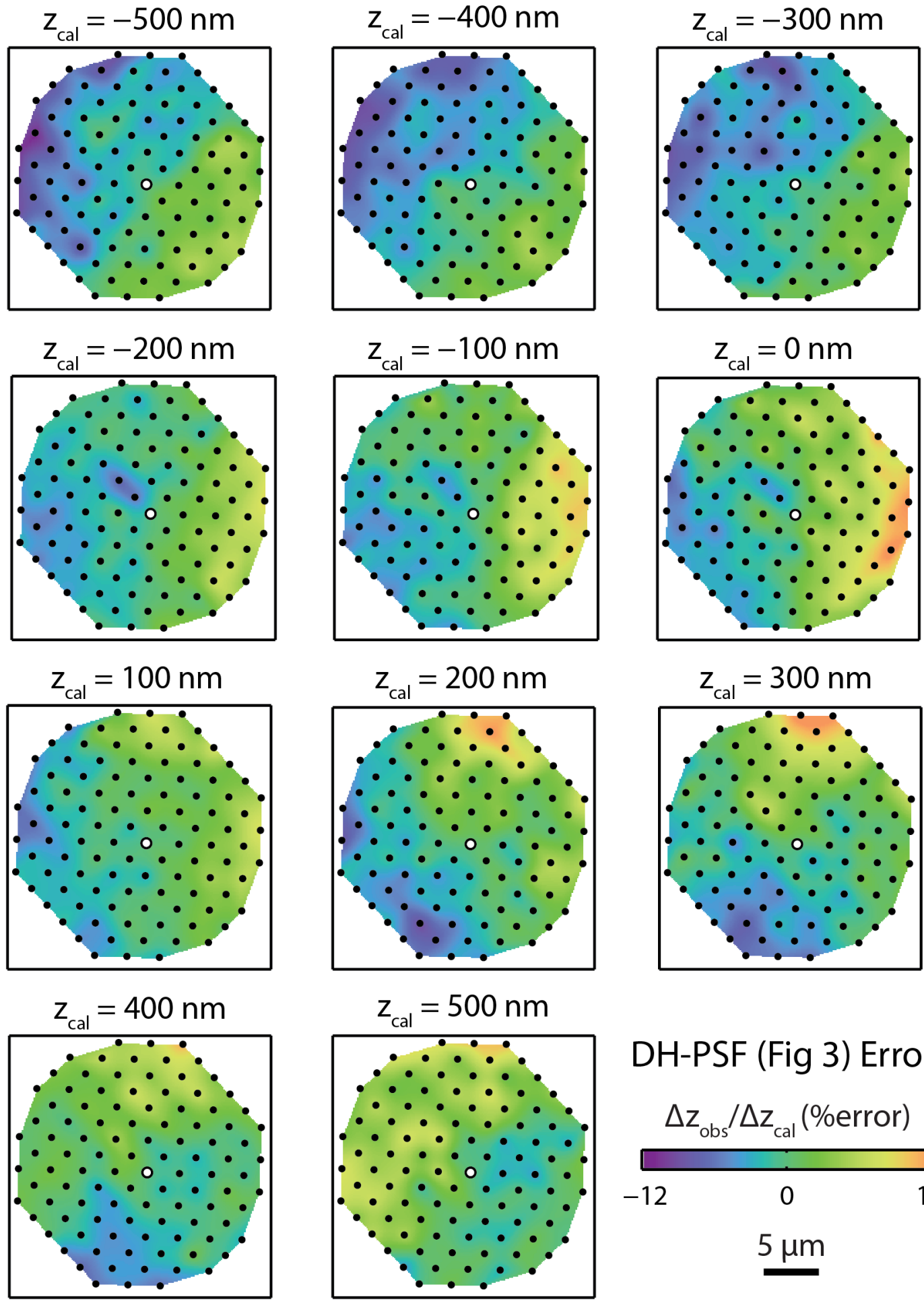

DH-PSF (Fig 3) Errors

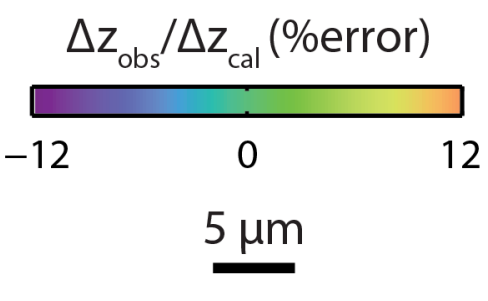

Figure S3. The measured errors in "3D response" $\Delta z_{o b s} / \Delta z_{\text {cal }}$ shown as fractional departure from unity i.e. $\left(\Delta z_{o b s}-\Delta z_{c a l}\right) / \Delta z_{\text {cal }}$, for the DH-PSF setup of Figure 3 estimated at many $z_{\text {cal }}$ positions. The nanohole used for calibration is marked in white. 


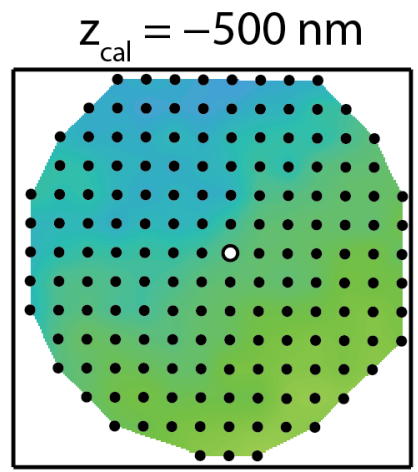

$z_{\mathrm{cal}}=-200 \mathrm{~nm}$
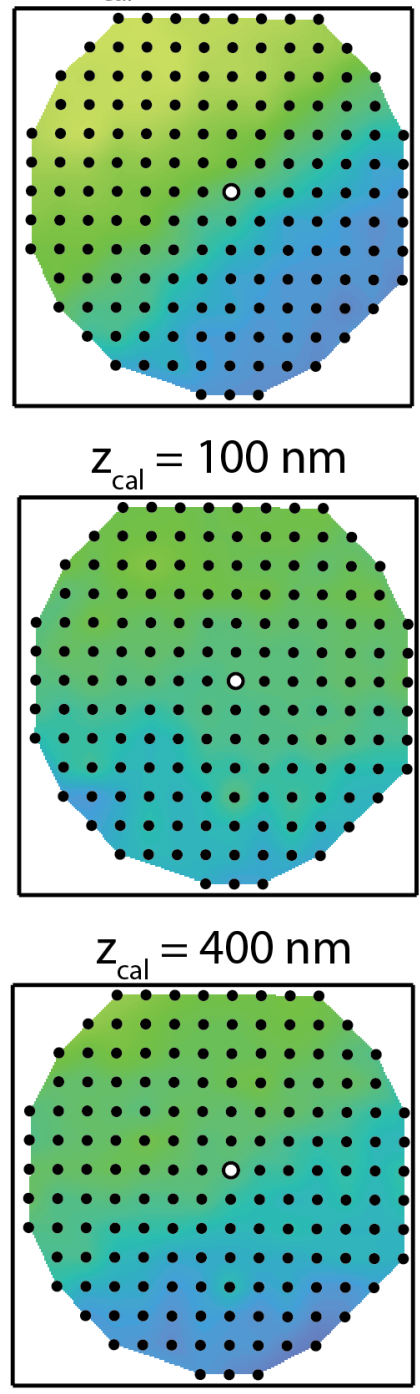
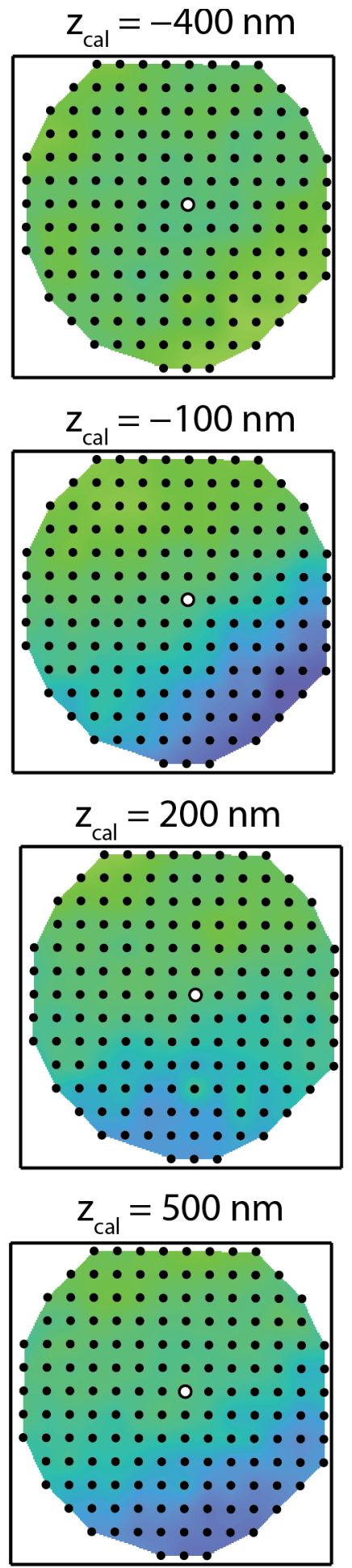
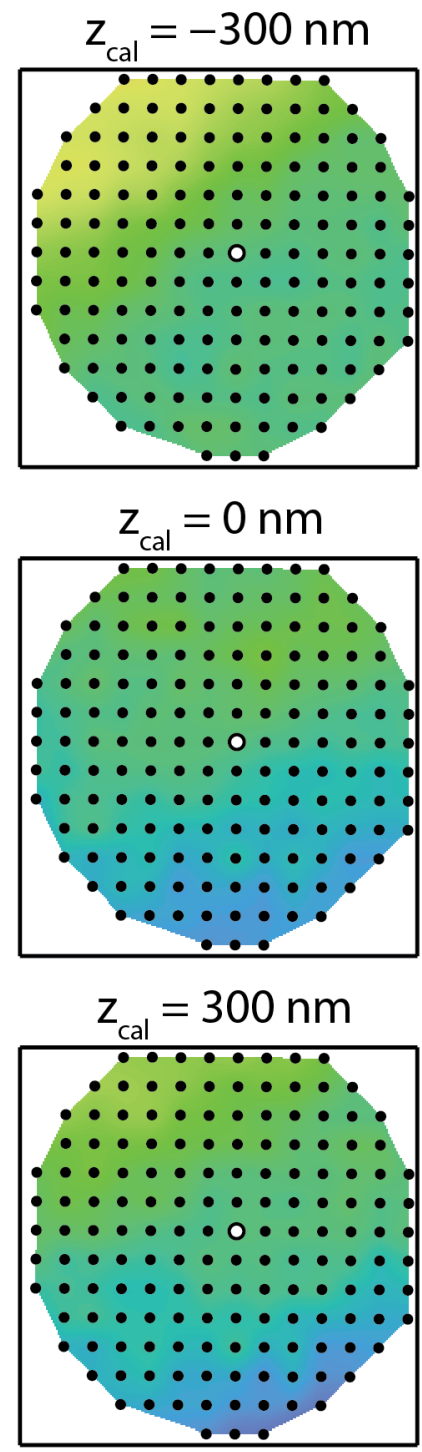

DH-PSF (Fig 4) Errors

$$
\frac{\Delta \mathrm{z}_{\mathrm{obs}} / \Delta \mathrm{z}_{\mathrm{cal}}(\% \text { error })}{-30 \quad 0}
$$

Figure S4. The measured errors in "3D response" $\Delta z_{o b s} / \Delta z_{\text {cal }}$ shown as fractional departure from unity, i.e. $\left(\Delta z_{o b s}-\Delta z_{c a l}\right) / \Delta z_{c a l}$, for the setup of Figure 4, using a DH-PSF mask, estimated at many $z_{c a l}$ positions. The nanohole used for calibration is marked in white. 


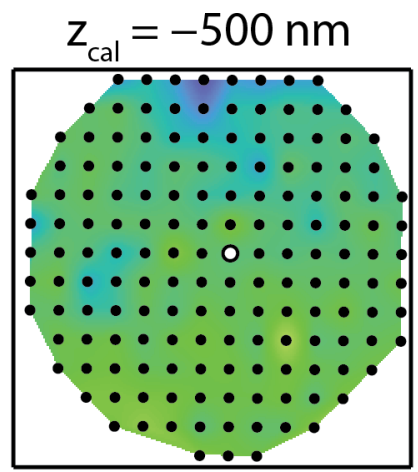

$\mathrm{z}_{\mathrm{cal}}=-200 \mathrm{~nm}$
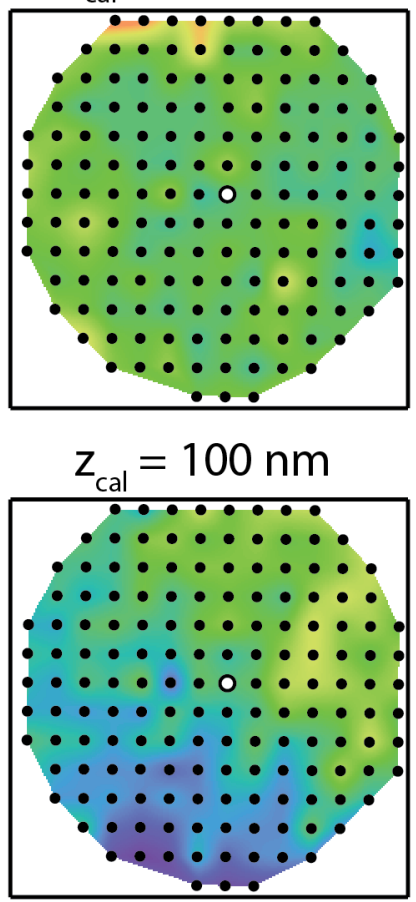

$\mathrm{z}_{\mathrm{cal}}=400 \mathrm{~nm}$

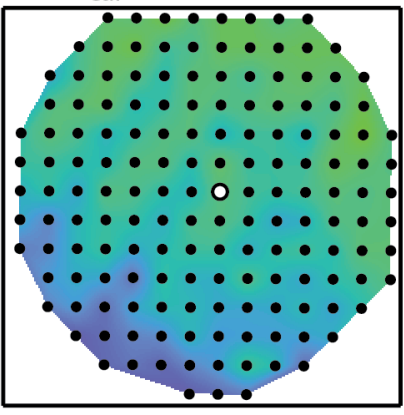

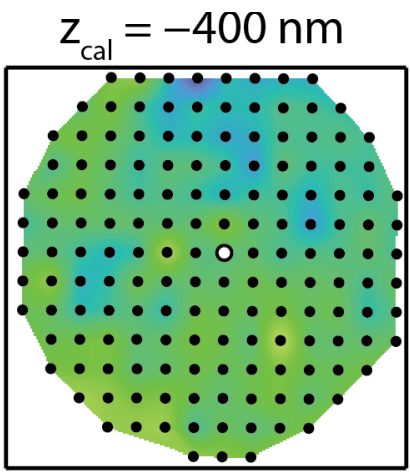

$\mathrm{z}_{\mathrm{cal}}=-100 \mathrm{~nm}$
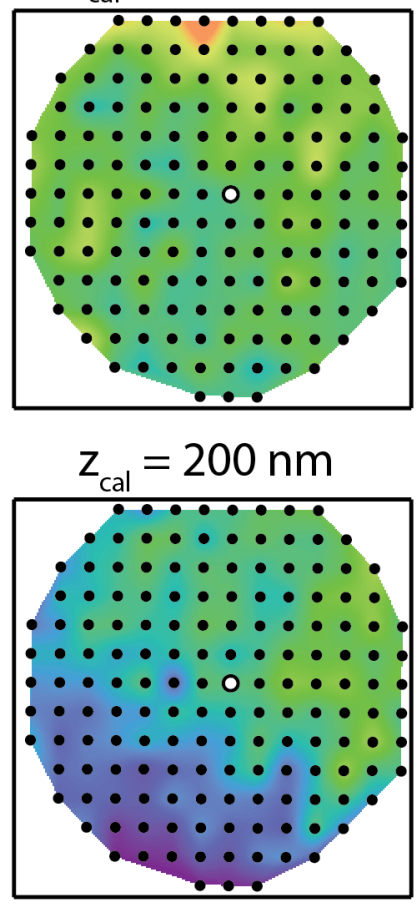

$\mathrm{z}_{\mathrm{cal}}=500 \mathrm{~nm}$

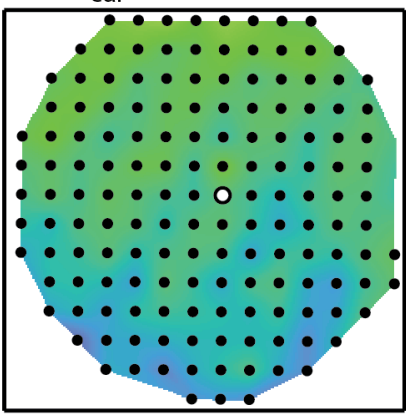

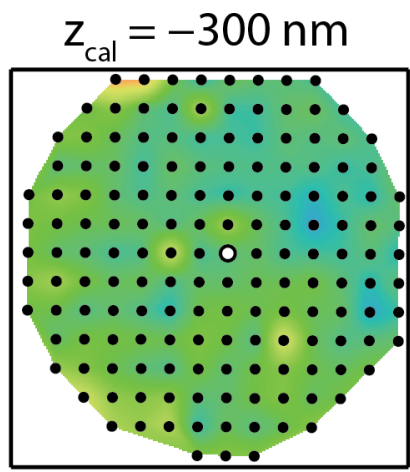
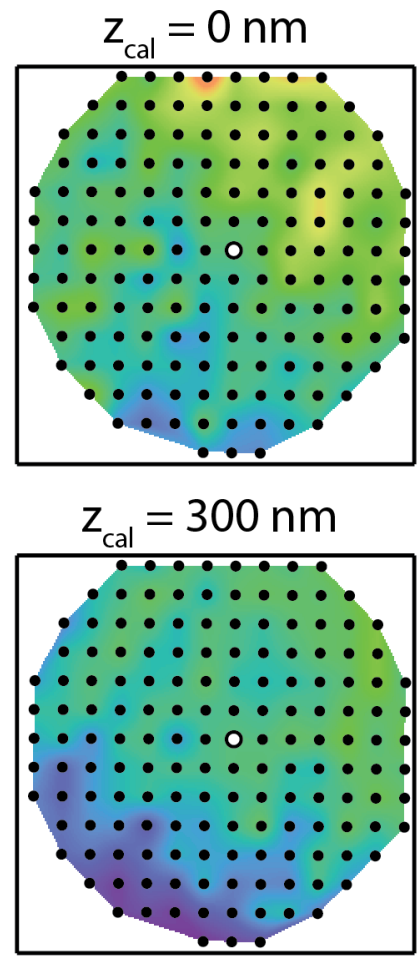

Astigmatism Errors

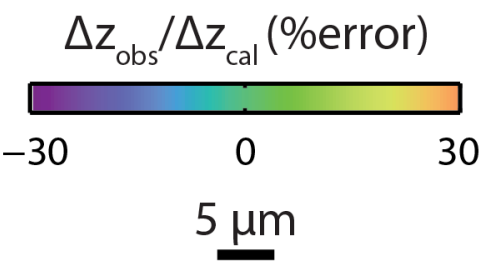

Figure S5. The measured errors in "3D response" $\Delta z_{o b s} / \Delta z_{\text {cal }}$ shown as fractional departure from unity, i.e. $\left(\Delta z_{o b s}-\Delta z_{c a l}\right) / \Delta z_{c a l}$, for the setup of Figure 4, using an astigmatic mask, estimated at many $z_{\text {cal }}$ positions. The nanohole used for calibration is marked in white. 


\section{DH-PSF errors}

\section{Tilt-corrected error}
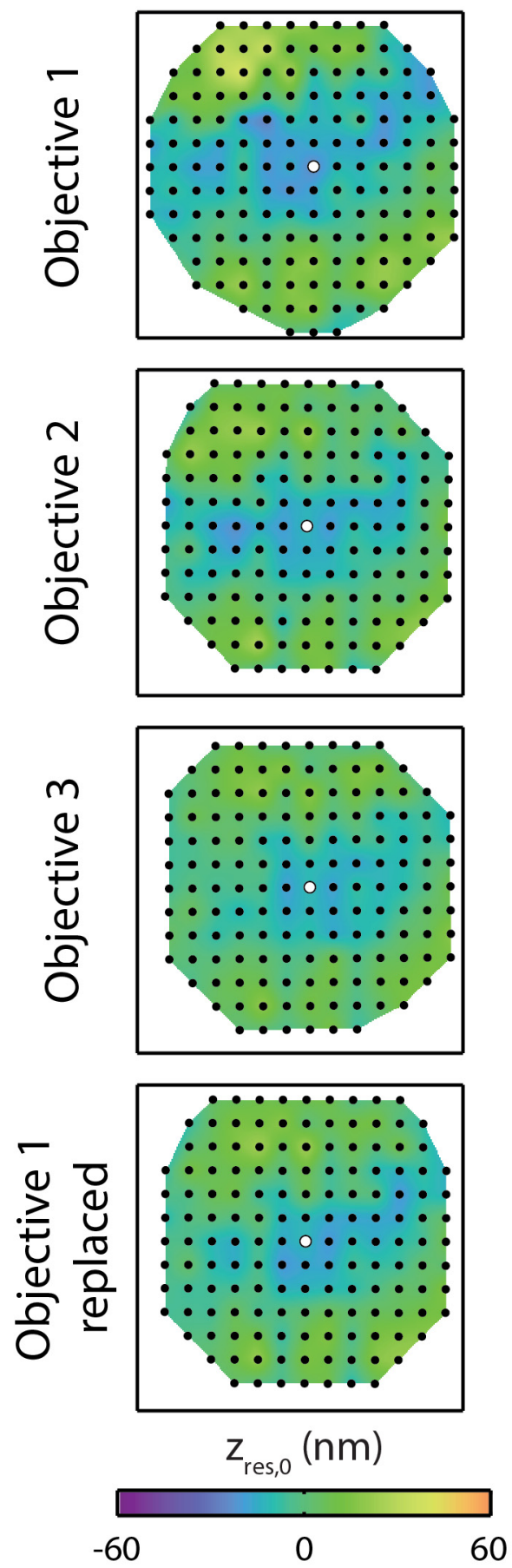

Response error, Response error,
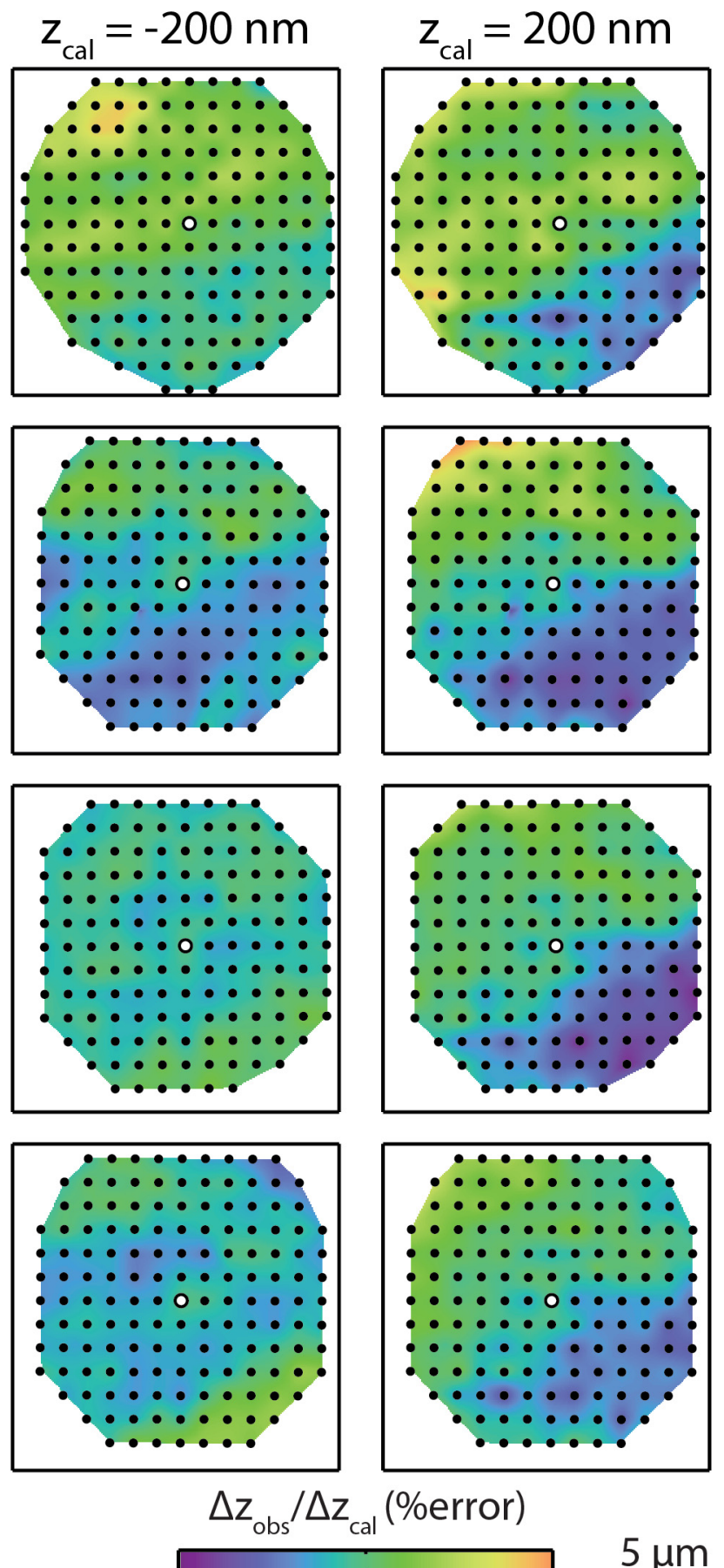

$5 \mu \mathrm{m}$

Figure S6. The effect of changing objectives and objective alignment on field-dependent errors in the DH-PSF. Shown are constant offset (corrected for planar tilt $z_{r e s, 0}$ ), and the response error $\Delta z_{o b s} / \Delta z_{c a l}$ shown as fractional departure from unity, i.e. $\left(\Delta z_{o b s}-\Delta z_{c a l}\right) / \Delta z_{c a l}$, for the DH-PSF using the same optical system as in Figure 4 . All objectives tested are the same make and model (Olympus UPLSAP0100X0). The measurement denoted "Objective 1 replaced" was taken after measurements using Objectives 1,2 , and 3 and reinserting Objective 1 into the optical system. The mounting of the objective lens into the microscope body generated slight variations in the response error, perhaps due to a small motion of the objective z-translator. The nanohole used for calibration is marked in white. The colormap of response error has been bounded to $\pm 15 \%$ to maximize contrast for the selected $z_{\text {cal }}$ positions; note this is different in Figure S7. 


\section{Astigmatism errors}

\section{Tilt-corrected error}
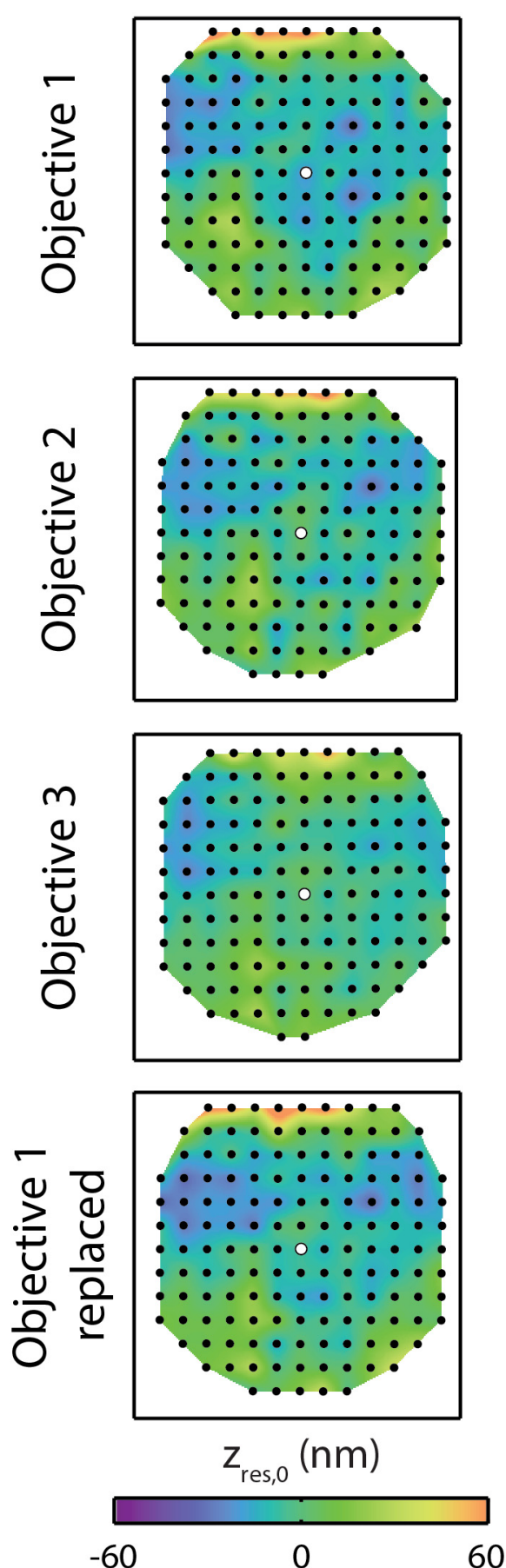

Response error, Response error,
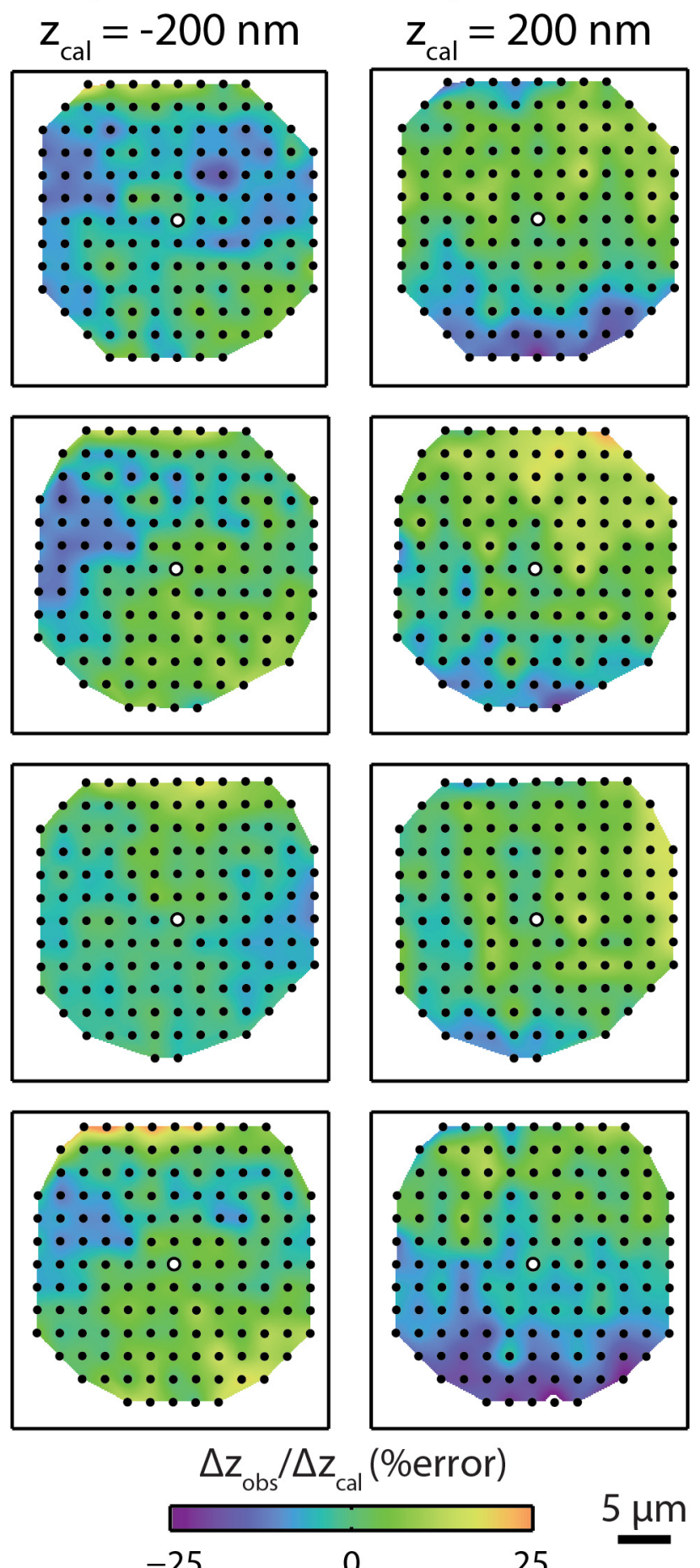

Figure S7. The effect of changing objectives and objective alignment on field-dependent errors in the astigmatic PSF. Shown are constant offset (corrected for planar tilt $z_{\text {res,o) }}$ ), and the response error $\Delta z_{o b s} / \Delta z_{c a l}$ shown as fractional departure from unity, i.e. $\left(\Delta z_{o b s}-\Delta z_{c a l}\right) / \Delta z_{\text {cal }}$, for the astigmatic PSF using the same optical system as in Figure 4. All objectives tested are the same make and model (Olympus UPLSAPO100XO). The measurement denoted "Objective 1 replaced" was taken after measurements using Objectives 1, 2, and 3 and reinserting Objective 1 into the optical system. The mounting of the objective lens into the microscope body generated slight variations in the response error, perhaps due a small motion of the objective z-translator. The nanohole used for calibration is marked in white. The colormap of response error has been bounded to $\pm 25 \%$ to maximize contrast for the selected $z_{\text {cal }}$ positions; note this is different in Figure S6. 

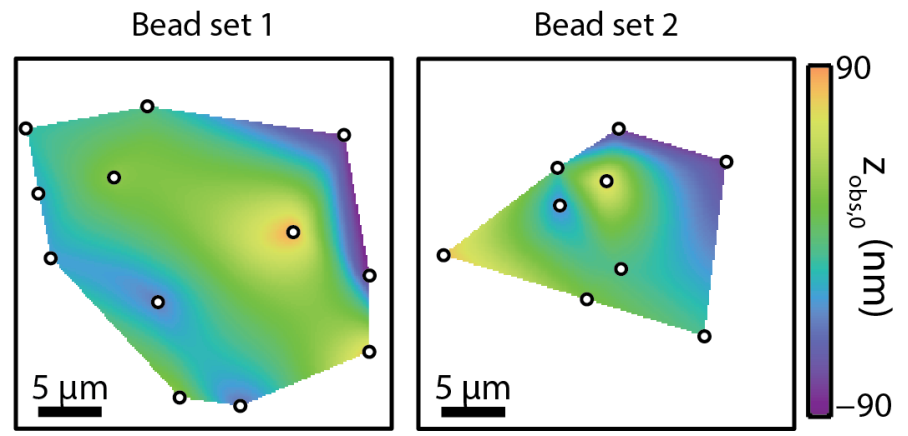

Figure S8. The variation in observed bead z positions when in focus $\left(Z_{o b s, 0}\right)$ for two example sets of fluorescent beads within the same sample. "Bead set 1 " is the one analyzed in Figure 5 . The z positions of beads were obtained by fitting DH-PSF images of beads with the global calibration. Any variation in $z_{o b s, 0}$ must result from a combination of bead-to-bead variability (either in the uniformity of dye attachment on the bead's surface, or true $z$ position in the 1\% PVA film) and field-dependent effects of the imaging system. Because the magnitude of the variation for beads is much larger than the variation for the nanohole arrays including apparent sample tilt [180 nm vs. $110 \mathrm{~nm}$, cf. Figures 3(a), 3(b)], and because the observed field-dependence of the pattern is not reproducible between sets of beads and changes rapidly throughout the field of view, we conclude that bead heterogeneity introduces significant variation in addition to that present in the imaging system. 